Vol. 50 (1994) [349-350]

\title{
Balanced block designs and various properties
}

\author{
AbDollah Khodkar
}

A block design is an ordered pair $(V, \mathcal{B})$, where $V$ is a finite set and $\mathcal{B}$ is a collection of subsets or multi-subsets of $V$ (called blocks). Usually extra conditions are imposed on this collection $\mathcal{B}$. In this work we study the existence of certain designs, the intersection problem for some designs, the structure of repeated blocks in designs and the existence of designs in which any two blocks have at most two elements in common (super-simple designs). We also investigate subsets of the element set of a design which intersect all the blocks of the design (covering sets). Finally, we study multi-subsets of the block set of a design which can be uniquely completed to the design.

We start in Chapter 1 with some necessary definitions. Then in Chapter 2 we deal with the construction of some designs called balanced ternary designs or BTDs. In Section 2.1 we first construct a family of cyclic BTDs of odd order $2 n-1$ with an automorphism of order $2 n-1$. Then in a similar way, we construct a BTD of order $n$ with block size $2 m$ for $4 \leqslant 2 m \leqslant n$. In Section 2.2 we give necessary and sufficient conditions for the existence of a BTD with block size 4 , any index $\lambda$, and $\rho_{2} \leqslant 6$. These results extend the results in Donovan [5] and [6].

In Chapter 3 we concentrate on the intersection problem for BTDs with block size 3. In Section 3.1 we give some general constructions for a BTD with a hole. Then in Sections 3.2, 3.3 and 3.4 we determine the number of common triples in two simple BTDs with block size 3 and index 2 , for $\rho_{2}=0,3$ and 4 . (Similar results for the cases $\rho_{2}=1,2$ can be found in Billington and Hoffman [1] and Billington and Mahmoodian [2].) In Section 3.5 we construct pairs of simple BTDs with block size 3 , index 3 and $\rho_{2}=3$ having exactly $m$ blocks in common for all admissible $m$. In Section 3.6 we investigate the intersection problem for directed triple systems with index 2. (A similar result for index 1 has appeared in Lindner and Wallis [10] and Fu [7].)

We study BTDs with repeated blocks in Chapter 4. Indeed, we determine the fine structure of a BTD with block size 3 , index 3 and $\rho_{2}=3$. This result is parallel to the result for balanced incomplete block designs or BIBDs with block size 3 and index 3 (see Colbourn, Mathon, Rosa and Shalaby [3] and Colbourn, Mathon and Shalaby [4]).

Received 31st March, 1994

Thesis submitted to The University of Queensland, November 1993. Degree approved: March 1994. Supervisor: Dr Elirabeth J Billington.

Copyright Clearance Centre, Inc. Serial-fee code: 0004-9729/94 \$A2.00+0.00. 
In Chapter 5 we study $\left(v ; \rho_{2} ; 4, \lambda\right)$ BTDs with the property that any 3-multisubset of the element set can occur at most once in the blocks of the design. Designs with this property are called super-simple designs. In Sections 5.2 and 5.3 we deal with super-simple $(v, 4,2)$ BIBDs, correcting a result in Gronau and Mullin [9]. In Sections $5.4,5.5$ and 5.6 we prove that there exists a super-simple $\left(v ; \rho_{2} ; 4, \lambda\right)$ BTD, where $\left(\rho_{2}, \lambda\right) \in\{(1,2),(2,2),(0,4)\}$, for all admissible $v$. Finally, in Section 5.7 , the non-existence of a certain super-simple group divisible design is proved.

In Chapter 6 we investigate smallest covering sets of $t$-designs. In Section 6.1, we give some preliminary results, including an upper bound on the size of the smallest covering set for a symmetric $(v, k, \lambda)$ BIBD. Then in Section 6.2 , we find some results on smallest covering sets for $t-(v, t+1, \lambda)$ designs. These results can be found in Gionfriddo and Lo Faro [8]. However our proofs are different. In Section 6.3 we give some interesting examples.

In Chapter 7 we find a smallest defining set for each of the 36 non-isomorphic two-fold triple systems of order nine.

\section{REFERENCES}

[1] E.J. Billington and D.G. Hoffman, 'Pairs of simple balanced ternary designs with prescribed numbers of triples in common', Austral. J. Combin. 5 (1992), 59-71.

[2] E.J. Billington and E.S. Mahmoodian, 'Multi-set designs and numbers of common triples', Graphs Combin. 9 (1993), 105-115.

[3] C.H. Colbourn, R.A. Mathon, A. Rosa and N. Shalaby, "The fine structure of threefold triple systems: $v \equiv 1$ or $3(\bmod 6)^{\prime}$, Discrete Math. $92(1991), 49-64$.

[4] C.H. Colbourn, R.A. Mathon and N. Shalaby, "The fine structure of threefold triple systems: $v \equiv 5(\bmod 6)$ ', Austral. J. Combin. 3 (1991), 75-92.

[5] D. Donovan, 'Balanced ternary designs with block size four', Ars Combin. 21 (1986), 71-88.

[6] D. Donovan, 'More balanced ternary designs with block size four', J. Statist. Plann. Inference 17 (1987), 109-133.

[7] H.L. Fu, 'Directed triple systems having a prescribed number of triples in common', Tamkang J. Math. 14 (1983), 85-90.

[8] M. Gionfriddo and G. Lo Faro, 'On blocking sets in $S_{d}(t, t+1, v)$ ', Mitt. Math. Sem. Giessen (1991), 55-58.

[8] H.-D.O.F. Gronau and R.C. Mullin, 'On super-simple $2-(v, 4, \lambda)$ designs', J. Combin. Math. Combin. Comput. 11 (1992), 113-121.

[10] C.C. Lindner and W.D. Wallis, 'Embeddings and prescribed intersections of transitive triple systems', Ann. Discrete Math. 15 (1982), 265-272. 